\title{
One Stage Reconstruction of Esophageal Atresia and Distal Tracheoesophageal Fistula in a 3250-gm Neonate: A Case Report
}

\author{
Bijay Upadhyay, Xuedong Wu*, Jun Li, Ning Wang, Shanshan Zhang, Na Li \\ Department of Pediatric Surgery, Affiliated Hospital of Dali University, Dali, China \\ Email: ${ }^{*}$ xuedong3288@sina.com
}

Received 28 April 2015; accepted 31 May 2015; published 2 June 2015

Copyright (C) 2015 by authors and Scientific Research Publishing Inc.

This work is licensed under the Creative Commons Attribution International License (CC BY). http://creativecommons.org/licenses/by/4.0/

(c) (i) Open Access

\section{Abstract}

Esophageal atresia (EA) occurs when the upper part of the esophagus does not connect with the lower part of esophagus and stomach. Tracheoesophageal fistula (TEF) is an abnormal connection between the upper part of the esophagus and the trachea. Treatment for esophageal atresia has advanced over several decades due to improvements in surgical techniques and neonatal intensive care. The aim is to share our experience regarding the treatment of esophageal atresia with tracheoesophageal fistula. A 4-day-old neonate suffering from esophageal atresia with type IIIB tracheoesophageal fistula underwent one stage esophageal reconstruction and obtained good outcome without any complications. In this paper, a simple intra-operative technique for tracheal fistula repair and end to end esophageal anastomosis is discussed. We used a simple technique that we have found useful for ligation of tracheal fistula. Anastomosis of lower and upper esophagus without any complication like anastomotic leakage or stricture/stenosis of the neonate with EA/TEF (type IIIB), was proved to be safe and effective.

\section{Keywords}

Esophageal Atresia, Tracheoesophageal Fistula, Esophageal Reconstruction, End to End Anastamosis

\section{Introduction}

Esophageal atresia (EA) with or without tracheoesophageal fistula (TEF) is a fairly common congenital disorder that doctor should consider in the differential diagnosis of a neonate who develops feeding difficulties and res-

\footnotetext{
*Corresponding author.
}

How to cite this paper: Upadhyay, B., Wu, X.D., Li, J., Wang, N., Zhang, S.S. and Li, N. (2015) One Stage Reconstruction of Esophageal Atresia and Distal Tracheoesophageal Fistula in a 3250-gm Neonate: A Case Report. Open Journal of Pediatrics, 5, 147-150. http://dx.doi.org/10.4236/ojped.2015.52022 
piratory distress in the first few days of life. It is often associated with other congenital anomalies, most commonly congenital cardiac defects. EA/TEF is a life threatening condition as well as esophagus reconstruction for the patients, prompt recognition, appropriate clinical management to prevent aspiration, and swift referral to an appropriate tertiary care center have resulted in a significant improvement in the rates of morbidity and mortality in these infants over the past 50 years. But the leakage of anastomosis and esophageal stenosis are still the main complications after operation. A neonate with EA and TEF underwent one stage reconstruction and who got good outcome was reported in this paper and the complications prevention management was discussed.

\section{Case Report}

The patient was 4-day-old, 3250 gm, female, born after 39 weeks and 6 days gestational age by uncomplicated primary lower segmental cesarean section. Because vomiting and respiratory distress after birth, progressive abdominal distention was noticed, a nasogastric tube could not be passed into the stomach and esophagogram was made through injected iodine oil via nasogastric tube given, contrast not visible under the level of 4th thoracic vertebra, forming an enlarged blind end and gas intra-alimentary tract can be found, the diagnosis of EA/ TEF with gross type III was established (Figure 1(a) and Figure 1(b)) smooth round pouch like structure and coiling of Ryle's tube seen (Figure 1). No other anomalies exist through total physical examination and investigations; surgical Correction was scheduled for the baby treatment.

General anesthesia was given without muscle relaxant and then an endotrecheal tube was inserted and gradually pushed until breath sounds can be heard both left and right lungs means the tube was in its right position. Then the patient was placed into the left lateral position. Horizontal S-shaped incision was given at the 6th intercostal space, thoracic cavity was opened and posterior mediastinum was exposed, the azygos vein was divided and cut down to reveal the underlying EA/TEF. The blind end of esophagus and the fistula were found and made label respectively (Figure 2).

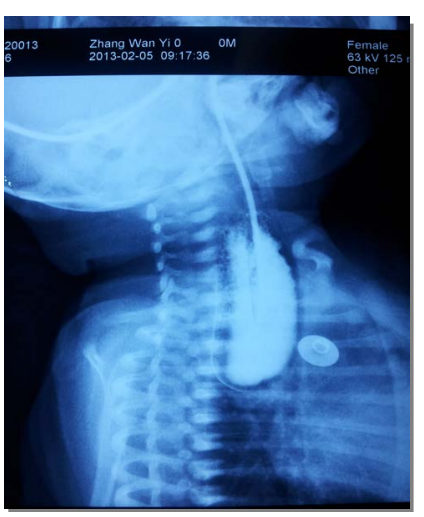

(a)

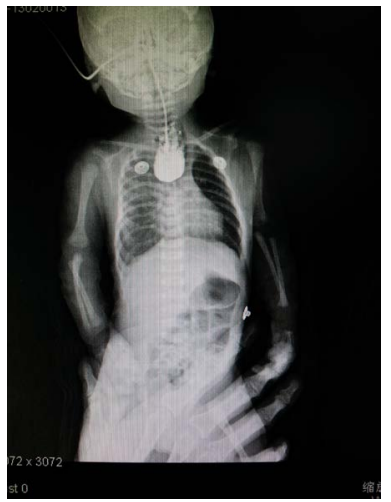

(b)

Figure 1. (a) Lateral view; (b) A/P view. Both X-ray esophagogram shows contrast not visible beyond the level of T4 vertebrae.

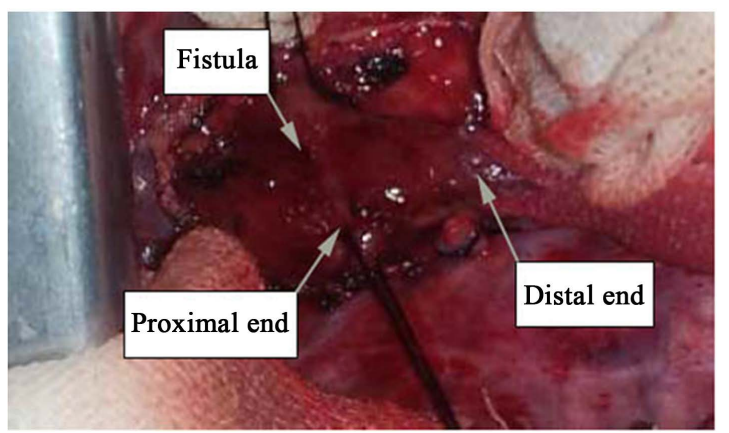

Figure 2. Operative photography showing the blind end of esophagus and fistula and labeled them respectively. 
The TEF was dissected circumferentially and its attachment to the membranous portion of trachea is taken down. The tracheal fistula opening and definition with diameter $0.5 \mathrm{~cm}$ was ligated and the end to tracheal was closed with nonabsorbable suture but the connective tissue between two pouches was not cut down completely. The gap between two ends was $1.5 \mathrm{~cm}$, the proximal esophagus pouch was mobilized as high as possible to afford a tension free esophageal anastomosis, The blood supply to the upper esophageal pouch is generally robust and is based on arteries derived from the thyrocervical trunk. However, the blood supply to lower esophagus is more tenuous and segmental, originating from intercostal vessels. As such, significant mobilization of the lower esophagus is avoided to prevent ischemia at the esophageal anastamosis.

After opened the proximal end the anastomosis was performed by interrupted suturing the posterior mucosal layer first with 6 - 0 absorbable vircryl and then Ryle's tube was inserted into stomach and anterior mucosal layer was sutured and then outer layer was sutured to give strength to the anastamosis with 6 sutures, connective tissue around the esophagus was not separated apart so that it can give strength and release the tension of anastamosis. No active bleeding in operative field and a chest tube was inserted at the seventh intercostal space to the right posterior axillary line. Incision was sutured layer by layer.

Surgical process went well with less bleeding and effective anesthesia. Patient was sent back to ward when awake and endotrecheal tube was put out. Antibiotics coverage and fluids management was done post operation and recovery was smoothly and uneventfully. Nasogastric tube was removed 2 weeks after operation and feed milk normal, contrast chest $\mathrm{x}$-ray with iodine oil feed shows normal chest and esophagus through with no leakage (Figure 3(a)). Patient was discharged after 16 days of operation. Contrast (iodine oil) chest x-ray followed up at 3 months after operation with no abnormal constriction and stenosis noted (Figure 3(b)) and the patient weight was $5000 \mathrm{gm}$. Up to now, the patient was followed-up for 2 years regularly without any swallowing difficulty and any other complains.

\section{Discussion}

Esophageal artesia with tracheoesophageal fistula is a life threatening condition occurs in one of 3000 to 5000 births with a complicated prognosis. Although survival in patients of EA with/without TEF has markedly improved due to advances in surgical techniques and neonatal intensive care, complications often occur and morbidities after treatment are still regarded as an important issue. The reasons for high morbidity associated with esophageal atresia are well known [1]-[3]. Associated anomalies, low birth weight or prematurity, and likelihood of pneumonia are common findings with esophageal artesia and affect treatment outcome. In addition, variations in the type of Artesia, anatomical differences, anastomotic tension induced by long gap of esophageal atresia and lack of surgical experience are also considered as potential risk.

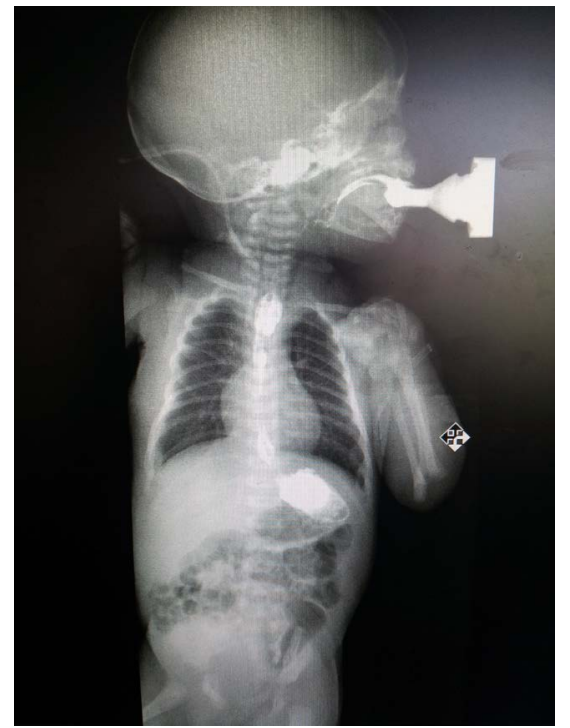

(a)



(b)

Figure 3. Both esophagogram shows no leakage and stenosis, contrast freely flowed to the stomach. 
Doctors who care for neonates should be aware of both the clinical presentation and management of neonates with these conditions. It may be suspected prenatally if ultrasound examination reveals polyhydramnios, absence of a fluid-filled stomach, a small abdomen. Lower than expected fetal weight and distended esophageal pouch. Fetal MRI may be used to confirm the presence of EA/TEF. EA may be detected postnataly by failure to pass nasogastric tube and radiographs that demonstrate coiling of the NG tube in the pouch, tracheal compression and deviation on plain chest radiographs, absence of a gastric bubble on plain radiographs, which may suggest EA without a TEF or EA with a proximal TEF. Three dimensional CT scanning, administration of contrast into the esophagus followed by chest radiographs can confirm the diagnosis.

As soon as the diagnosis is made patient need to be prepared for prompt surgical treatment for optimal outcome in which it's out-comes are mainly depends on the surgery. Treatments of EA have gradually evolved due to not only better pre-operative and postoperative neonatal intensive care but also the application of meticulous and precise surgical technique. Recently, the mortality rate associated with EA was reported to be less than $10 \%$ and high risk neonates now have better prognosis [4].

Many procedures can be selected for esophageal repair with one or two stages according to different type of the malformation, but anastomotic stricture or leak are still both of the most common complications after operation. The suture material, type of anastomosis, anastomotic tension, ischemia, anastomotic leak and the presence of GER are the main factors affecting the development of anastomotic strictures [4]-[7]. The technique intraoperation and the way of anastomosis are very important for post-operative complications preventing.

As in this case, EA/TEF, type III, one stage reconstruction was performed immediately after clinical diagnosis was established. The distal esophageal segment was less mobilized due to the risk of damage to the connective tissue and occult arterial supply. This was imperative to prevent additional damage to the arterial supply, so as to prevent ischemia that may lead to anastomosis leakage. The ansatomosis was performed using single layer of mucosa to mucosa anastomosis technique with interrupted absorbable sutures. The Ryle's tube was inserted into the stomach after the posterior mucosal layer was sutured. Then only anterior mucosal layer was sutured. The outer adventitia layer was strengthened with few non absorbable sutures. The Ryle's tube was kept in situ for 2 weeks. This technique was found to be effective for anastomotic strictures and leakage prevention. As a result, no anastomotic leakage and uneventful recovery was achieved in the post-operative period. Regular 2 years follow up showed no complain with deglutition.

\section{Conclusion}

In this case, we used a simple technique by sparing the connective tissue around the esophagus and single layer mucosa to mucosa anastomosis technique. We have found this useful for end to end esophageal anastomosis. Ligation of tracheal fistula with nonabsorbable sutures was also very effective. Anastomosis of lower and upper esophagus without any complication of anastomotic leakage or stricture/stenosis of the neonate with EA/TEF (type IIIB), was proved to be safe and effective.

\section{References}

[1] Lopez, P.J., Keys, C., Pierro, A., et al. (2006) Oesophageal Atresia: Improved Outcome in High-Risk Groups? Journal of Pediatric Surgery, 41, 331-334. http://dx.doi.org/10.1016/j.jpedsurg.2005.11.009

[2] Castilloux, J., Noble, A.J. and Faure, C. (2010) Risk Factors for Short- and Long-Term Morbidity in Children with Esophageal Atresia. Journal of Pediatrics, 156, 755-760. http://dx.doi.org/10.1016/j.jpeds.2009.11.038

[3] Townsend, B., et al. (2012) The Biological Basis of Modern Surgical Practice. 2, 1837-1839.

[4] Spitz, L. (2007) Oesophageal Atresia. Orphanet Journal of Rare Diseases, 2, 24. http://dx.doi.org/10.1186/1750-1172-2-24

[5] Singh, S.J. and Shun, A. (2001) A New Technique of Anastomosis to Avoid Stricture Formation in Oesophageal Atresia. Pediatric Surgery International, 17, 575-577. http://dx.doi.org/10.1007/s003830100579

[6] Chang, A.C. and Orringer, M.B. (2007) Management of the Cervical Esophagogastric Anastomotic Stricture. Seminars in Thoracicand Cardiovascular Surgery, 19, 66-71. http://dx.doi.org/10.1053/j.semtcvs.2006.11.001

[7] Upadhyaya, V.D., Gangopadhyaya, A.N., Gupta, D.K., et al. (2007) Prognosis of Congenital Tracheoesophageal Fistula with Esophageal Atresia on the Basis of Gap Length. Pediatric Surgery International, 23, 767-771. http://dx.doi.org/10.1007/s00383-007-1964-0 